\title{
RECONSTRUCTION OF NONSTATIONARY SIGNALS ALONG THE WAVELET RIDGES
}

\author{
NALAN ÖZKURT \\ Electrical and Electronics Engineering Department, \\ Dokuz Eylul University, Izmir, Turkey \\ nalan.ozkurt@eee.deu.edu.tr \\ F. ACAR SAVACI \\ Electrical and Electronics Engineering Department, \\ Izmir Institute of Technology, Izmir, Turkey \\ acarsavaci@iyte.edu.tr
}

Received May 28, 2004; Revised January 14, 2005

\begin{abstract}
In this paper, the nonstationary signals have been recovered using the skeleton along the wavelet ridges in the noisy case and the attractors of the cleaned signals are reconstructed in the phase space by time-delay embedding. In order to verify the signal recovery and reconstruction procedure, the similarity measure Hausdorff distance between cleaned, noise-free original attractors have been calculated. The computations show that the procedure reduces the noise level acceptably and the reconstructed attractors are more similar to the original attractors.
\end{abstract}

Keywords: Nonstationary signals; chaotic attractors; wavelet ridges; reconstruction.

\section{Introduction}

Since the time-domain methods and frequency domain methods alone, do not provide adequate information about the nonlinear dynamical systems, the time-frequency domain methods have been attracting many researchers. The wavelets [Wong \& Chen, 2001; Ozkurt \& Savac1, 2001; Chandre et al., 2003], the Wigner-Ville distribution [Chen, 1994; Lima \& Cohen, 1998; Galleani et al., 1999] have been used to analyze chaotic systems. The wavelet transform describes the timevariation of the spectral content of the signal with its multiresolution property. Therefore, the frequency components of the nonstationary signals may be resolved more accurately with wavelet analysis. The time-frequency domain representation of a single trajectory contains relevant information about the phase space structures while the asymptotical quantities such as Lyapunov exponents, fractal dimension and entropy only reflect the asymptotical behavior [Chandre et al., 2003].
However, it is difficult to interpret the wavelet analysis results especially for complex signals. In this case, the information about the signal can be extracted along the ridges of the wavelet transform and the signal can be recovered by using wavelet transform coefficients along the ridges which are called skeleton of the wavelet transform [Delprat et al., 1992]. The wavelet ridge extraction methods such as stationary phase method and the simple method have been introduced in [Delprat et al., 1992] but they do not yield the actual ridges for contaminated signals accurately. The method for extracting the ridges of the contaminated signals developed by [Carmona et al., 1997, 1999] is quite accurate but it is not efficient in computational cost. Therefore, the new ridge detection method based on the singular value decomposition (SVD) has been proposed and the wavelet ridges of the contaminated signals have been determined by the SVD-based ridge determination method in [Ozkurt \& Savac1, 2005]. 


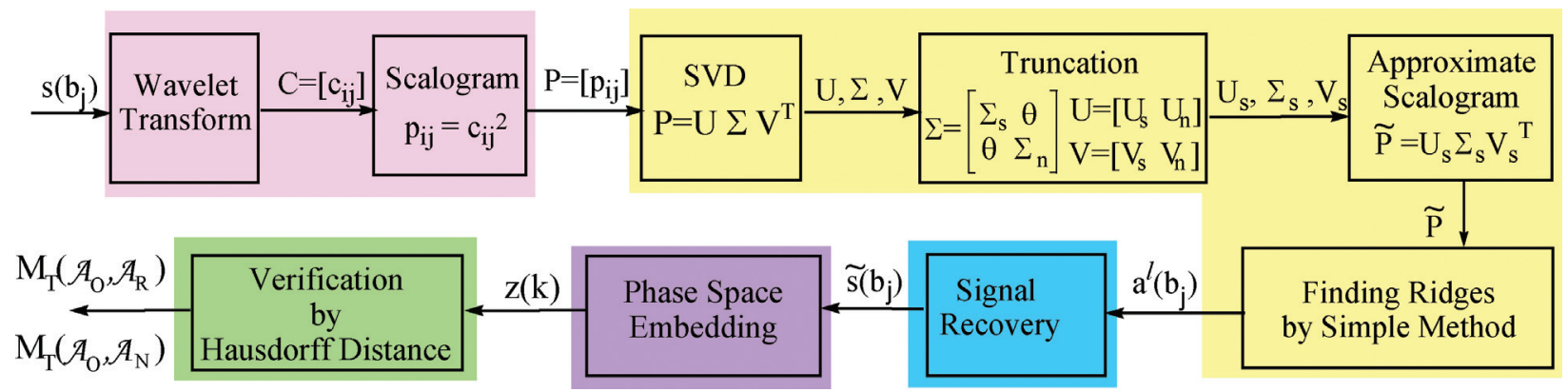

Fig. 1. The block diagram of the reconstruction of the nonstationary signal along the wavelet ridges.

In the present paper, the attractors of the chaotic signals have been reconstructed in the phase space by using the time-delay embedding of the signal obtained from wavelet ridges. In order to determine the accuracy of the attractor reconstruction and the noise reduction procedure, the Hausdorff distances between the noise-free original attractor and these attractors have been calculated. The wavelet ridges of the noisy chaotic signals have been calculated by the ridge determination algorithm proposed in [Ozkurt \& Savac1, 2005]. It has been shown that the Hausdorff distance between the original attractor of the noise-free signal and the attractor of the cleaned signal is smaller than the Hausdorff distance between the noise-free original attractor and the attractor of the contaminated signal.

In the second section of this paper, the properties of the continuous wavelet transform and the wavelet ridges have been briefly given and the determination of the wavelet ridges for the noisy signals [Ozkurt \& Savac1, 2005] is introduced. In Sec. 3, the recovery of the nonstationary signals along the wavelet ridges, the phase space reconstruction of the recovered signal and the test for accuracy of the approximation using Hausdorff distance have been given as the main contribution of this paper. The proposed method has been summarized in Fig. 1 in which the pink and the yellow parts have already been discussed in [Ozkurt \& Savac1, 2005]. In Sec. 4, this new procedure has been applied to the spiral attractor and the double-scroll attractor of Chua's circuit in order to verify the proposed method.

\section{Determination of Wavelet Ridges and Noise Reduction}

Before explaining the determination of the wavelet ridges and noise reduction procedure, some necessary definitions about the wavelet transform and the wavelet ridges will be given in the sequel.

\subsection{Wavelet transform and wavelet ridges}

The integral wavelet transform of a signal $s(t)$ is defined as

$$
W_{s}(a, b ; \Psi) \triangleq \int_{-\infty}^{\infty} s(t) \Psi_{a, b}^{*}(t) d t
$$

where $\Psi(\cdot)$ is called as "mother wavelet" satisfying the admissibility condition [Mallat, 1999], and * denotes the complex conjugate, and $a$ and $b$ are the dilation (scale) and translation coefficients, respectively. The scaled and translated wavelet is obtained as

$$
\Psi_{a, b}(t) \triangleq \frac{1}{a} \Psi\left(\frac{t-b}{a}\right), \quad a \in \mathbb{R}^{+}, \quad b \in \mathbb{R} .
$$

The local time-frequency energy density which is called scalogram $P_{s}(a, b ; \Psi)$ has been defined in the wavelet domain as in [Mallat, 1999]

$$
P_{s}(a, b ; \Psi) \triangleq\left|W_{s}(a, b ; \Psi)\right|^{2}
$$

The continuous wavelet transform conserves the total energy of the signal $E_{T}$ according to the Plancherel's formula as in [Delprat et al., 1992]

$$
\begin{aligned}
E_{T} \triangleq\|s\|_{2}^{2} & =\int_{-\infty}^{\infty}|s(t)|^{2} d t \\
& =c_{\psi}^{-1} \int_{-\infty}^{\infty} \int_{0}^{\infty}\left|W_{s}(a, b ; \Psi)\right|^{2} \frac{d a d b}{a}
\end{aligned}
$$

where $c_{\psi}$ is the admissibility constant.

For the numerical computations, the discrete samples of the continuous wavelet transform have been considered and the scaled and translated 
wavelets have been defined at the dyadic grid as

$$
\Psi_{m, n}(t)=a_{0}^{-m / 2} \Psi\left(a_{0}^{-m} t-n b_{0}\right) \quad m, n \in \mathbb{Z}
$$

where $a_{0}, b_{0} \in \mathbb{R}$ are the dilation and translation coefficients, respectively.

If the wavelets defined in Eq. (5) are chosen so as to constitute a Riesz basis for every $s(t) \in L^{2}(\mathbb{R})$, by considering only finite number of basis vectors for practical purposes, the total energy in Eq. (4) can be approximately given in [Ozkurt \& Savac1, 2005] as

$$
\tilde{E}_{T} \triangleq \frac{k}{c_{\psi}} \sum_{m=1}^{M} \sum_{n=1}^{N} p_{m n}
$$

where $k \triangleq b_{0} \ln a_{0}$ and the entries of the scalogram matrix $P=\left[p_{m n}\right]_{M \times N}$ are defined as

$$
p_{m n} \triangleq\left|c_{m n}\right|^{2}
$$

which are the local time-frequency domain energy densities evaluated at the discrete dilations $a_{m}$ and translations $b_{n}$. The truncation of the infinite dimensional scalogram matrix in time has been carried out by observing the attractor of the chaotic signal in the phase space with sufficient number of the samples.

The energy distribution of a wide class of signals at a particular time is concentrated at more than one frequency in the time-frequency plane along the wavelet ridges. The ridges are the curves at the time-frequency plane along which the energy is locally maximum. The relevant information about the signal can be obtained by extracting the wavelet transform coefficients along the ridges, which are called the skeleton of the wavelet transform.

The multicomponent signal with the instantaneous amplitudes $A_{l}(t)$ and the instantaneous phases $\phi_{l}(t)$ can be described by

$$
s(t)=\sum_{l=1}^{L} A_{l}(t) e^{j \phi_{l}(t)}
$$

where $L$ is the number of components, then the wavelet transform can be written as in [Delprat et al., 1992; Carmona et al., 1999]

$$
W_{s}(a, b ; \Psi)=\frac{1}{2} \sum_{l=1}^{L} A_{l}(b) e^{j \phi_{l}(b)} \hat{\Psi}^{*}\left(a \phi_{l}^{\prime}(b)\right)+r(a, b)
$$

where $r(a, b) \sim O\left(\left|A_{l}^{\prime}\right|,\left|\phi_{l}^{\prime \prime}\right|\right)$

where the prime denotes the derivative. Therefore, if the Fourier transform of the mother wavelet " $\hat{\Psi}(\omega)$ " is localized near a certain frequency $\omega=\omega_{0}$, the scalogram is localized around $L$ curves

$$
a^{l}=a^{l}(b)=\frac{\omega_{0}}{\phi_{l}^{\prime}(b)}, \quad l=1, \ldots, L
$$

which are called the ridges of the wavelet transform.

\subsection{Noise reduction by SVD based ridge determination}

When the signal is noise-free, the wavelet ridges can be determined simply by calculating the local maxima. However, in the noisy case some additional operations are required. In case of additive white Gaussian noise, the wavelet coefficients related with the noise are distributed all over the time-frequency plane and added to the wavelet coefficients related to the original signal, due to the linearity of the wavelet transform. A wavelet ridge determination algorithm based on SVD for noisy signal has been proposed in [Ozkurt \& Savac1, 2005]. In this method, the scalogram of the noisy signal is obtained and SVD is applied to the scalogram matrix. Then, the approximate scalogram is obtained using only the dominant singular values of the scalogram. Finally, the ridges are determined by finding the local maxima of the approximated scalogram for each time instant. In the case of mono-component signal, since the signal has single instantaneous frequency at a given time instant, a single ridge (the main ridge) will be sufficient to locate the energy concentrations. SVD-based ridge determination and noise reduction procedure have been illustrated in the yellow part of the block diagram in Fig. 1.

\section{Signal Recovery, Phase Space Reconstruction and Verification}

After the wavelet ridges of the noisy signal have been determined, the signal can be recovered from the wavelet transform coefficients and the attractor of the chaotic signal is reconstructed in the phase space by time-delay embedding [Abarbanel, 1996]. The reconstructed attractor and the noisefree attractor are compared in the phase space in terms of Hausdorff distance.

\subsection{Signal recovery}

Since the skeleton of the wavelet transform contains the relevant information about the signal, the signal can be recovered using the wavelet coefficients along the ridge. The real part of the signal $s(t)$ 
given in Eq. (8) can be approximately reconstructed from the skeleton of the transform using the formula Eq. (9) by neglecting the correction term $r(a, b)$ and using the normalized mother wavelet $\hat{\Psi}^{*}\left(a^{l}(b) \phi_{l}^{\prime}(b)\right)=\hat{\Psi}^{*}\left(\omega_{0}\right)=1$ as

$$
\tilde{s}(b)=2 \operatorname{Re}\left\{\sum_{l=1}^{L} W_{s}\left(a^{l}(b), b ; \Psi\right)\right\}
$$

where $L$ is the number of ridges. The signal recovery using the transform skeleton is a simple scheme and it produces good approximations. But all the points along the ridge must be included in the recovery [Carmona et al., 1999]. The signal recovery has been shown by the blue block in Fig. 1 .

\subsection{Phase space embedding}

The phase space can be reconstructed by time-delay embedding from the approximate discrete-time scalar signal according to Taken's Embedding theorem [Abarbanel, 1996; Kantz \& Schreiber, 2002] by properly choosing the embedding dimension and embedding delay.

Let a vector $z \in \mathbb{R}^{d}$ be reconstructed from the scalar observations " $s(k)$ " as

$$
z(k)=[s(k-T) s(k-2 T) \cdots s(k-d T)]
$$

where $d$ is the embedding dimension and $T$ is the embedding delay. By embedding, the reconstructed attractor in the phase space is plotted using the vector $z(k)$ such that there is one-to-one mapping between the reconstructed attractor and the original attractor which preserves the information about the derivatives of the original flow [Kantz \& Schreiber, 2002; Kostelisch \& Schreiber, 1993].

In the reconstruction of the embedded vector, the first minimum of average mutual information is chosen as the time delay and the embedding dimension is selected using "False Nearest Neighborhood Method". The Nonlinear Dynamics Toolbox [Reiss] is used for the selection of the embedding dimension and the embedding delay. The purple block in Fig. 1 represents the phase space embedding of the recovered signal.

\subsection{Verification}

The approximation can be accepted as accurate if the distance between the attractors of the original signal and the reconstructed signal is less than the distance between the attractors of the original signal and the contaminated signal as

$$
h\left(\mathcal{A}_{O}, \mathcal{A}_{R}\right)<h\left(\mathcal{A}_{O}, \mathcal{A}_{N}\right)
$$

where $\mathcal{A}_{O}, \mathcal{A}_{R}$ and $\mathcal{A}_{N}$ are the attractors of the original, reconstructed and contaminated signals, respectively, and $h(\mathbf{A}, \mathbf{B})$ is the Hausdorff distance which can be classified as the similarity measure [Veltkamp, 2001].

Definition 3.1. Let $(\mathbf{X}, d)$ be a complete metric space, and $\mathcal{H}(\mathbf{X})$ denote the space whose points are the compact subsets of $\mathbf{X}$, other than the empty set. Let $\mathbf{A}, \mathbf{B} \in \mathcal{H}(\mathbf{X})$, then the Hausdorff distance between two points $\mathbf{A}$ and $\mathbf{B}$ is defined by

$$
h(\mathbf{A}, \mathbf{B}) \triangleq \max (\mu(\mathbf{A}, \mathbf{B}), \mu(\mathbf{B}, \mathbf{A}))
$$

where $\mu(\mathbf{A}, \mathbf{B}) \triangleq \max _{a \in A}\left(\min _{b \in B}(d(a, b))\right)$ and $d(a, b)$ is the usual Euclidian distance between points $a \in \mathbf{A}$ and $b \in \mathbf{B}$ [Barnsley, 1993].

Remark. Although the signals in the time domain are quite different due to a shift in time and/or mean value, and/or a change in frequency, the approximation may be considered as successful if the original and reconstructed attractors are similar in the phase space. While the shift in time or a change in frequency cannot be observed in the phase space, the shift in the mean value has been seen as the translation of the attractor in the phase space. Therefore, instead of the usual Hausdorff distance, the Hausdorff distance under translations of the objects, $M_{T}(\cdot, \cdot)$ which is defined below, has been selected as the similarity measure.

Definition 3.2. The minimum Hausdorff distance between all possible relative positions of the two sets is defined as

$$
M_{T}(\mathbf{A}, \mathbf{B}) \triangleq \min _{t \in T} h(\mathbf{A}, t \mathbf{B})
$$

where $T$ is the group of translations [Huttenlocher et al., 1993].

\section{Applications}

The recovery of nonstationary signals from the contaminated measurements using the ridge detection algorithm based on the singular value decomposition method and the phase space reconstruction is accomplished for the spiral attractor and doublescroll attractors of the Chua's circuit.

In the applications, the wavelet transform is demonstrated by choosing the following standard 
complex Morlet mother wavelet

$$
\Psi(t)=\frac{1}{\sqrt{2 \pi}} \cdot e^{j \omega_{0} t} e^{-t^{2} / 2}
$$

where $\omega_{0}$ defines the center frequency of the wavelet.

The dynamics of the Chua's circuit is described by the following differential set of equations

$$
\begin{aligned}
& \dot{x}=\alpha(y-h(x)), \\
& \dot{y}=x-y+z, \\
& \dot{z}=-\beta y .
\end{aligned}
$$

where $\alpha$ and $\beta$ are the parameters defined by the circuit components and the piecewise linear characteristics are given as $h(x)=\left(m_{1}-m_{0}\right)$ $(|x+1|-|x-1|)$ where $m_{1}=-1 / 7, m_{0}=-2 / 7$ [Chua, 1992].

\subsection{Spiral attractor}

The wavelet transform of the state variable $x(t)$ with additional Gaussian white noise of variance $\sigma^{2}=0.2$ is numerically computed for the spiral attractor which is observed for $\alpha=8.50000425$ and $\beta=14.28$ in Chua's circuit. The number of scales is $M=120$ and the time instants $N=500$. The scalogram of the contaminated state variable $x(t)$ of the spiral attractor is shown in Fig. 2(a).

The approximate scalogram $\tilde{\mathbf{P}}$ is obtained by reducing the noise in selecting the singular values of which the energy contribution is greater than $10 \%$ of the total energy. Then the wavelet ridges are determined for the spiral attractor. The wavelet ridges are shown in Fig. 2(b).

The signal $x(t)$ is recovered by using Eq. (11) along the determined ridges and the phase space is reconstructed using Taken's embedding theorem [Abarbanel, 1996]. The 2D projection of original, contaminated and the reconstructed attractors in the $x-y$ plane has been illustrated in Fig. 3 .

The Hausdorff distance between the set of the points in the numerically computed noisefree and reconstructed spiral attractors has been calculated for scanning the phase space for all possible translations of the reconstructed attractor. The minimum distance in Eq. (15) has been obtained as $M_{T}\left(\mathcal{A}_{O}, \mathcal{A}_{R}\right)=0.2265$. The Hausdorff distance between the original attractor and the contaminated attractor $\mathcal{A}_{N}$ has been calculated as $M_{T}\left(\mathcal{A}_{O}, \mathcal{A}_{N}\right)=1.0364$.

\subsection{Double-scroll attractor}

The double-scroll attractor is observed for $\alpha=9$ and $\beta=14.28$ in Chua's circuit. The normalized scalogram is obtained for the state variable $x(t)$ with additional Gaussian white noise with variance $\sigma^{2}=0.2$. The normalized scalogram matrix is calculated for $M=120$ scales and $N=1000$ time instants. The scalogram matrix is shown in Fig. 4(a).

The approximate scalogram $\tilde{\mathbf{P}}$ is obtained by choosing the dominant singular values which have energy contribution greater than $10 \%$. Then the wavelet ridges which are shown in Fig. 4(b), are calculated and the phase space reconstructed. The embedding dimension $d=3$ and the time delay $T=7 \tau_{s}$ have been selected by nonlinear dynamics Toolbox [Reiss]. The projection of the attractors to $x-y$ plane is shown in Fig. 5 .

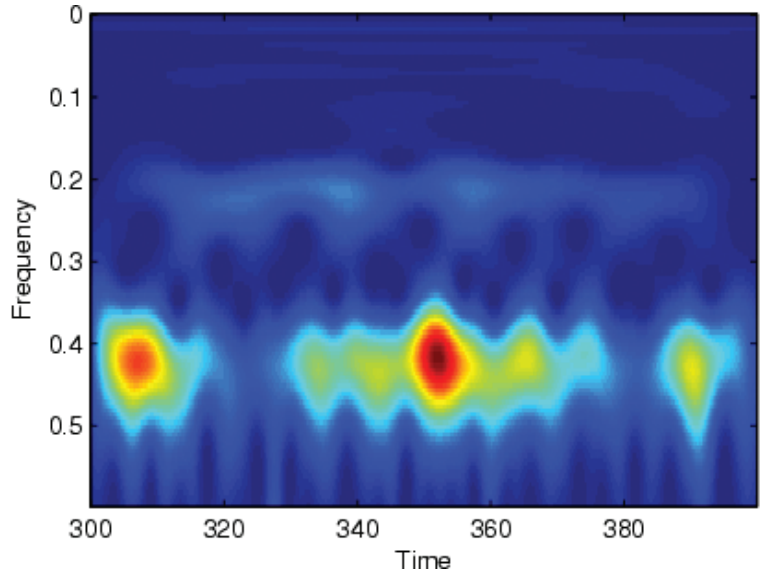

(a)

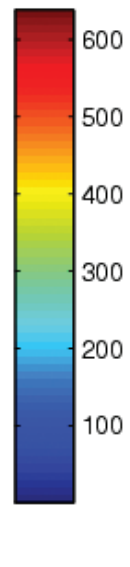

Fig. 2. (a) Scalogram and (b) the ridges of the spiral attractor.

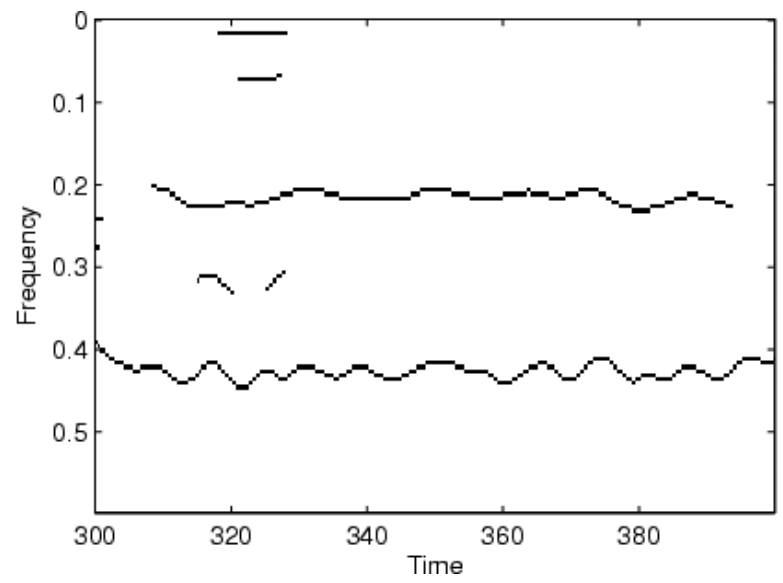

(b) 


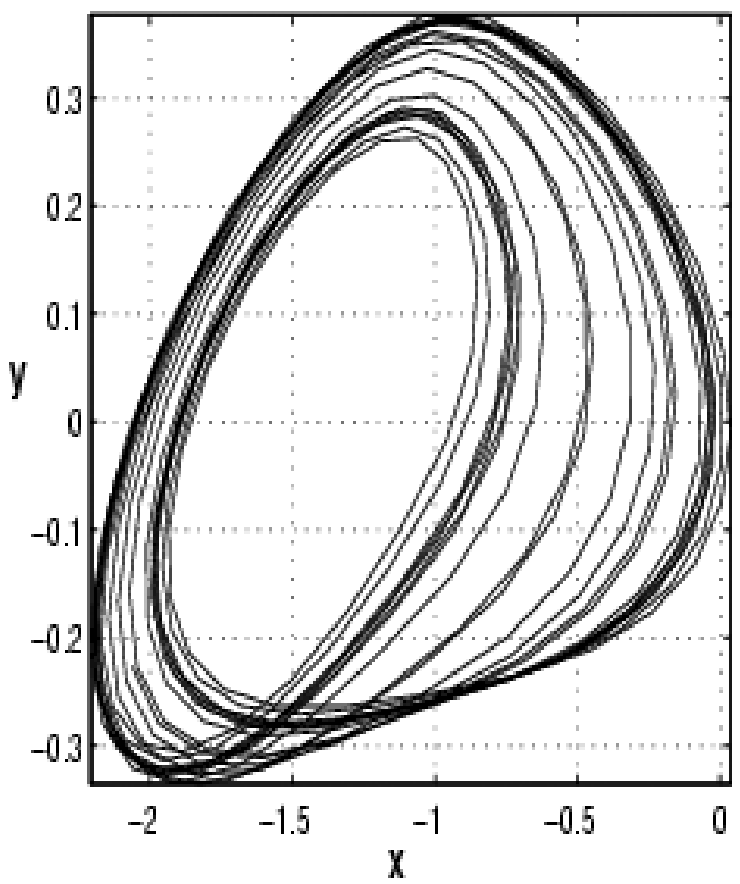

(a)

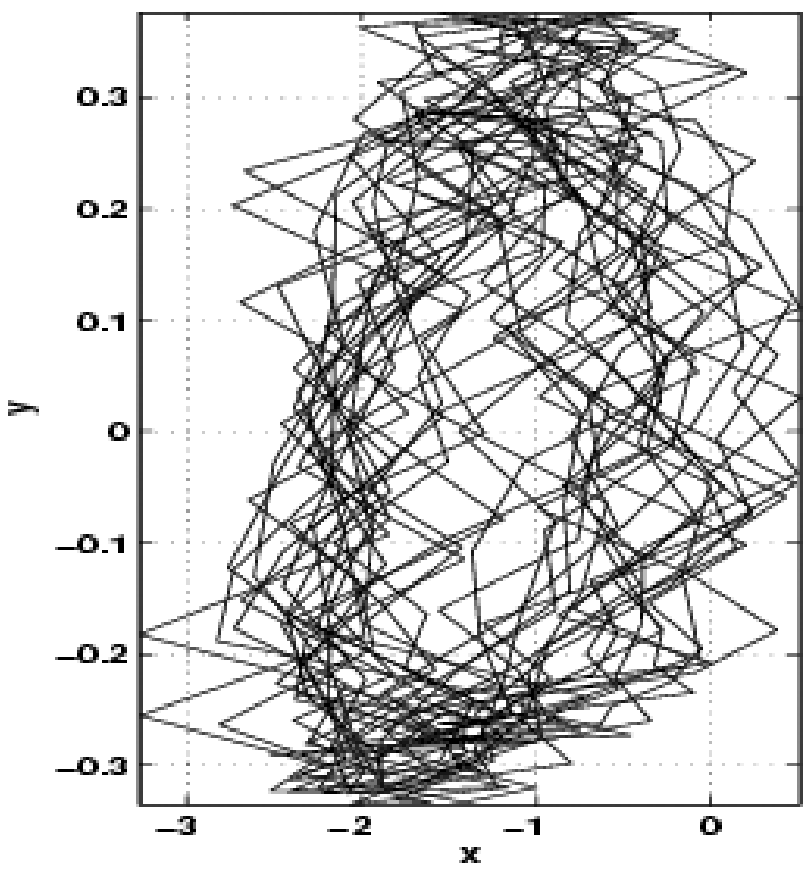

(b)

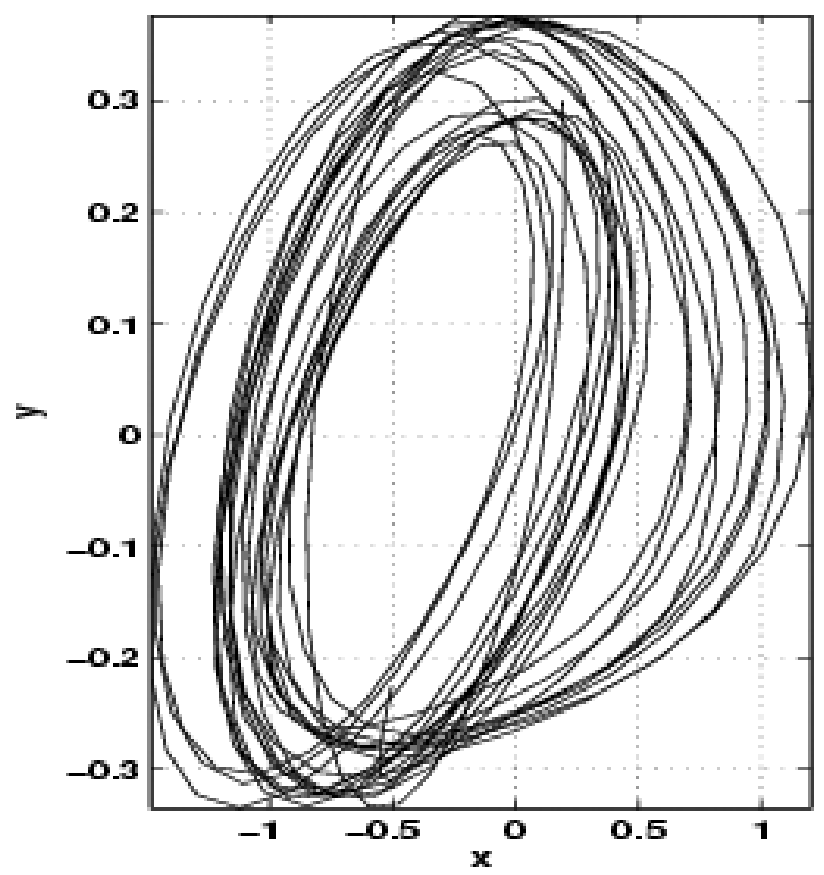

(c)

Fig. 3. The attractors of (a) original, (b) contaminated, (c) reconstructed signals for spiral attractor, respectively.

The minimum Hausdorff distance between the numerically computed noise-free attractor and the reconstructed attractor, and the distance between the noise-free and contaminated attractor have been calculated as

$$
M_{T}\left(\mathcal{A}_{\mathcal{O}}, \mathcal{A}_{\mathcal{R}}\right)=0.3862<M_{T}\left(\mathcal{A}_{\mathcal{O}}, \mathcal{A}_{\mathcal{N}}\right)=0.8366
$$




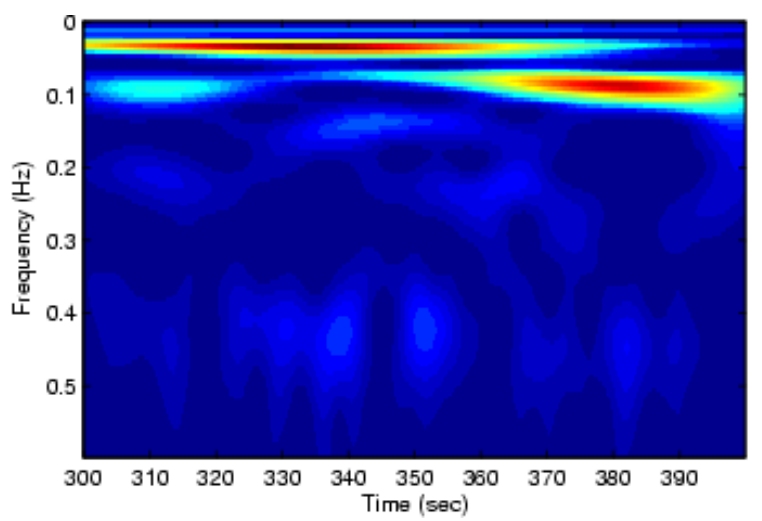

(a)

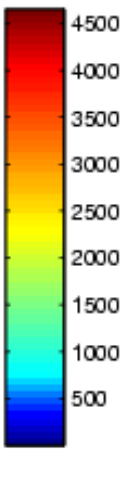

Fig. 4. (a) Scalogram, and (b) the ridges of the double-scroll attractor.

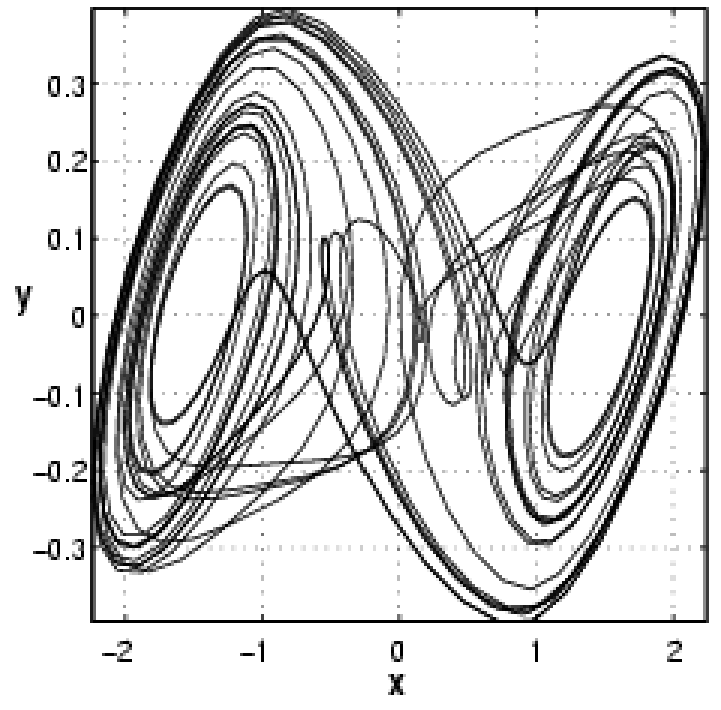

(a)

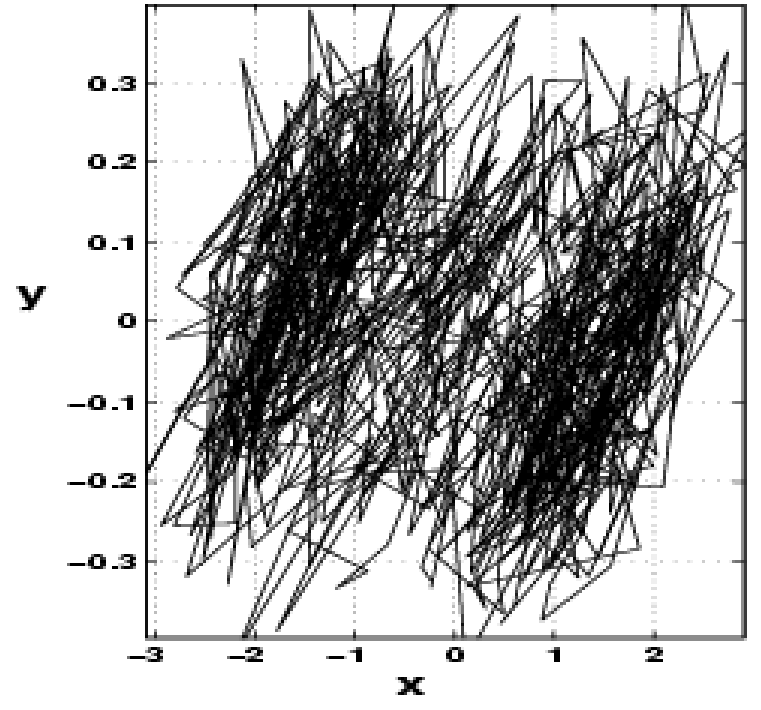

(b)

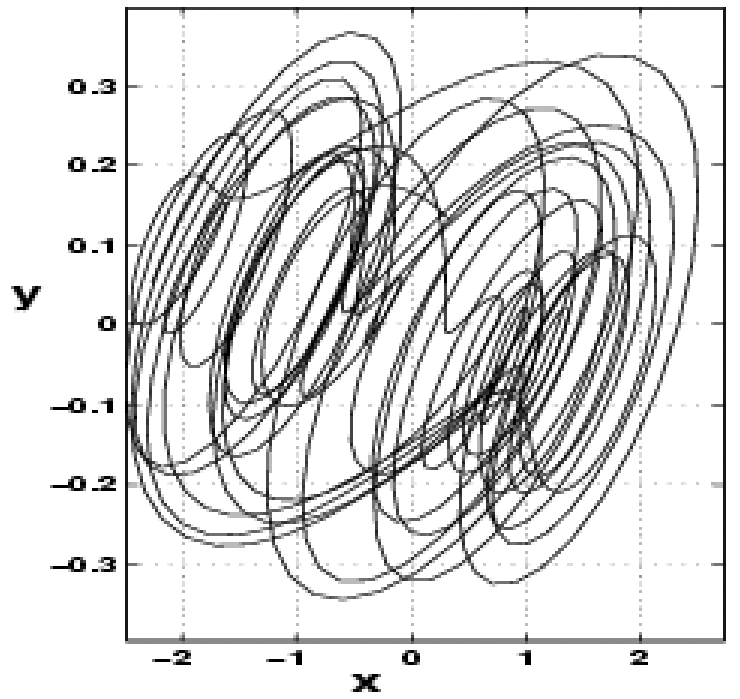

(c)

Fig. 5. The attractors of (a) original, (b) contaminated, (c) cleaned signals for double-scroll attractor, respectively. 


\section{Conclusions}

The reconstruction of the attractors of the Chua's circuit from the contaminated attractors has been accomplished using wavelet domain characteristics. The signal has been analyzed in the wavelet domain and the ridges of the transform have been obtained using SVD based ridge determination method. Then, the signal is recovered from the wavelet domain coefficients along the ridges of the state variable $x(t)$, and the attractors embedded in the phase space. Finally, the minimum Hausdorff distance between the original and reconstructed attractors and the distance between the original and noisy attractors have been calculated. The computations show that the Hausdorff distances have reduced $78 \%$ for spiral attractor and $54 \%$ for double-scroll attractor.

\section{References}

Abarbanel, H. D. I. [1996] Analysis of Observed Chaotic Data (Springer, NY).

Barnsley, M. F. [1993] Fractals Everywhere (Academic Press, CA).

Carmona, R. A. \& Hwang, W. L. [1997] "Characterization of signals by the ridges of their wavelet transforms," IEEE Trans. Sign. Process. 45, 25862590.

Carmona, R. A., Hwang, W. L. \& Torresani, B. [1999] "Multiridge detection and time-frequency reconstruction," IEEE Trans. Sign. Process. 47, 480-492.

Chandre, C., Wiggins, S. \& Uzer, T. [2003] "Timefrequency analysis of chaotic systems," Physica $D$ 181, 171-196.

Chen, P. [1994] "Study of chaotic dynamical systems via time-frequency analysis," Proc. IEEE-SP Int. Symp. Time-Frequency and Time-Scale Analysis, pp. 357360.

Chua, L. O. [1992] "The genesis of Chua's circuit," $A E U$ 46, 250 .

de Moor, B. [1988] Mathematical Concepts and Techniques for Modelling of Static and Dynamic Systems, $\mathrm{PhD}$ Dissertation, Katholieke University, Leuven.

Delprat, N., Escudie, B., Guillemain, P., KronlandMartinet, R., Tchamitchian, P. \& Torresani, B. [1992]
"Asymptotic wavelet and Gabor analysis: Extraction of instantaneous frequencies," IEEE Trans. Inform. Th. 38, 644-664.

Galleani, L., Biey, M., Gilli, M. \& Presti, L. [1999] "Analysis of chaotic signals in the time-frequency plane," IEEE Int. Conf. Nonlinear Signal and Image Processing NSIP99, pp. 100-104.

Golub, G. H. \& Van Loan, C. F. [1996] Matrix Computations (Johns Hopkins University Press).

Huttenlocher, D. P., Klanderman, G. A. \& Rucklidge, W. J. [1993] "Comparing images using the Hausdorff distance," IEEE Trans. Patt. Anal. Mach. Intell. 15, 850-863.

Kantz, H. \& Schreiber, T. [2002] Nonlinear Time Series Analysis (Cambridge University Press, UK).

Kostelich, E. J. \& Schreiber, T. [1993] "Noise reduction in chaotic time-series data: A survey of common methods," Phys. Rev. E 48, 1752-1763.

Lima, E. \& Cohen, L. [1998] "Time-frequency analysis of harmonic oscillator motion," Proc. IEEE-SP Int. Symp. Time-Frequency and Time-Scale Analysis, pp. 25-28.

Mallat, S. [1999] A Wavelet Tour of Signal Processing, 2nd edition (Academic Press, San Diego, CA).

Ozkurt, N. \& Savaci, F. A. [2001] "Wavelet analysis of the generalized Chua's circuit," Proc. 9th Workshop on Nonlinear Dynamics of Electronic Systems 2001, the Netherlands, pp. 227-230.

Ozkurt, N. \& Savac1, F. A. [2005] "Determination of wavelet ridges of nonstationary signals by singular value decomposition method," IEEE Trans. Circuits Syst.-II: Express Briefs 52, 480-485.

Reiss, J., Nonlinear Dynamics Toolbox, http://www. physics.gatech.edu/

Todorovska, M. I. [2001] "Estimation of instantaneous frequency of signals using the continuous wavelet transform," Technical Report, http://www. usc.edu/dept/civil_eng/Earthquake_eng.

Veltkamp, R. C. [2001] "Shape matching: Similarity measures and algorithms," Technical Report, Utrecht University, the Netherlands.

Wong, L. A. \& Chen, J. C. [2001] "Nonlinear and chaotic behavior of structural system investigated by wavelet transform techniques," Int. J. Non-Lin. Mech. 36, 221-235. 